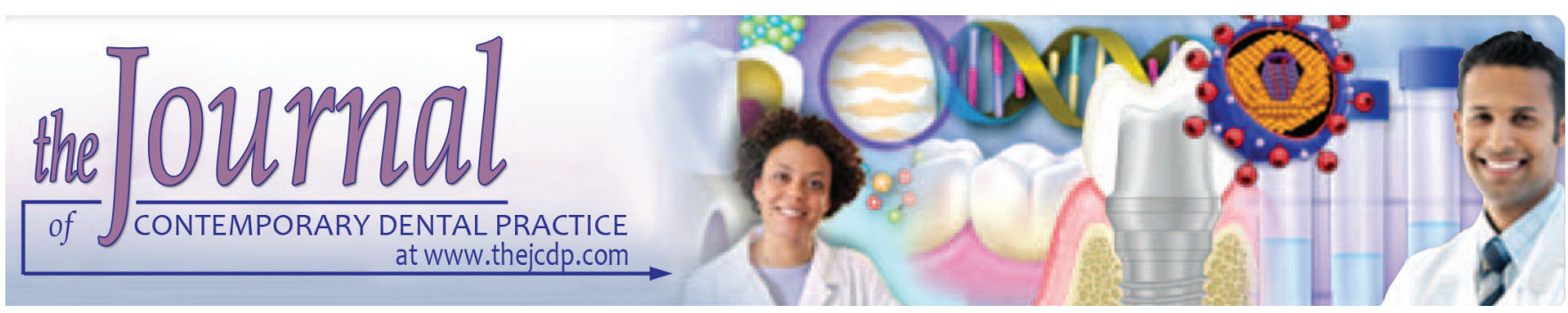

\title{
Comparison between Fluoride and Nano-hydroxyapatite in Remineralizing Initial Enamel Lesion: An in vitro Study
}

${ }^{1}$ Issa Daas, ${ }^{2}$ Sherine Badr, ${ }^{3}$ Essam Osman

\begin{abstract}
Aim: The aim of this study was to compare the effectiveness of nano-hydroxyapatite (nano-HAP) paste and fluoride varnish in remineralizing initial enamel lesion in young permanent teeth and their ability to resist secondary caries under dynamic $\mathrm{pH}$ cycling quantitatively and qualitatively.
\end{abstract}

Materials and methods: Initial caries-like lesions were artificially developed on 45 specimens. Specimens were divided into three groups: (1) Control (without treatment), (2) fluoride varnish (3M ESPE), and (3) nano-HAP paste (Desensibilize Nano $P$ ). The nano-HAP paste was applied twice separated by one $\mathrm{pH}$ cycle, and the varnish was applied only once followed by 7 days of $\mathrm{pH}$ cycling. All specimens were examined using DIAGNOdent ${ }^{\circledR}$ pen (KaVo, Germany), and a representative specimen was randomly selected from each group for qualitative evaluation using scanning electron microscope (SEM) at four stages: Baseline, after lesion formation, immediately after remineralization, and after $\mathrm{pH}$ cycling. Data were statistically analyzed with Statistical Package for the Social Sciences (SPSS), version 20.

Results: The degree of demineralization was significantly elevated in control group; however, no significant difference was found between fluoride varnish group and nano-HAP paste group $(p<0.001)$.

Conclusion: Nano-HAP paste showed promising long-term protective effect in terms of surface depositions and maintaining a smooth surface compared with fluoride varnish.

${ }^{1}$ Division of Pediatric Dentistry, Department of Developmental Sciences, Faculty of Dentistry, Beirut Arab University, Beirut Lebanon

${ }^{2}$ Division of Pediatric Dentistry, Department of Developmental Sciences, Faculty of Dentistry, Beirut Arab University, Beirut Lebanon; Department of Pediatric Dentistry and Public Health Cairo University, Giza, Egypt

${ }^{3}$ Department of Restorative Sciences, Faculty of Dentistry, Beirut Arab University, Beirut, Lebanon

Corresponding Author: Issa Daas, Division of Pediatric Dentistry, Department of Developmental Sciences, Faculty of Dentistry, Beirut Arab University, Beirut, Lebanon, Phone: +962791959718, e-mail: i.n.daas77@gmail.com
Clinical significance: Based on the findings of this study, nanoHAP paste might be recommended as alternative remineralizing agent with lower fluoride concentration than fluoride varnish that could be beneficial for children, pregnant females, and those who are at high risk of dental fluorosis.

Keywords: Enamel lesion, Nano-hydroxyapatite, Remineralization.

How to cite this article: Daas I, Badr S, Osman E. Comparison between Fluoride and Nano-hydroxyapatite in Remineralizing Initial Enamel Lesion: An in vitro Study. J Contemp Dent Pract 2018;19(3):306-312.

Source of support: Nil

Conflict of interest: None

\section{INTRODUCTION}

The modern dental practice is focusing on prevention and minimal intervention, and the replacement of lost tooth substance with biomimetic material is considered as one of the fundamentals of minimal intervention dentistry, with the goal of remineralizing an incipient lesion rather than extensive surgical replacement. Several methods were introduced to remineralize an early tooth structure loss. ${ }^{1,2}$

Fluoride varnishes have been known to be effective in the prevention of incipient enamel lesion in the presence of calcium and phosphate ions as it advances the development of fluorapatite. ${ }^{3}$ However, the adoption of remineralization of initial enamel lesions within the concept of minimal intervention dentistry led to the introduction of several agents and compounds as bioactive formulation of tricalcium phosphate, bioactive glass (calcium sodium phosphosilicate), casein-based products, and, finally, nano-HAP. ${ }^{4-6}$

The biomimetic nano-HAP has the ability to protect the teeth with the creation of a new layer of synthetic enamel around the tooth, rather than hardening the existing layer with fluoride. ${ }^{4}$ Several studies on nanoHAP as a biomimetic material showed the capability of 
remineralizing initial enamel lesions under $\mathrm{pH}$ cycling model. $^{7-9}$

The newly introduced nano-HAP paste is a bioactive agent that contains calcium nano-phosphate organized in a crystalline form of HA incorporated with highly concentrated sodium fluoride of 9000 ppm fluoride ion (Desensibilize Nano P, FGM, Joinville, Brazil). This paste is indicated for desensitization and/or remineralization of the enamel and is commercially available for professional use. According to the manufacturer, the maximum remineralizing effect can be observed by two professional applications of the paste, but the estimated number of sessions may vary according to the clinical judgment.

The purpose of this study was to evaluate the effect of fluoride varnish vs nano-HAP paste in remineralizing initial enamel caries quantitatively using quantitative laser fluorescence DIAGNOdent ${ }^{\circledR}$ pen (KaVo, Germany) and qualitatively using SEM. It was hypothesized that there is no difference between the effects of the two remineralizing agents on initial enamel caries.

\section{MATERIALS AND METHODS}

This controlled experimental in vitro study was conducted using intact human premolars extracted for orthodontic reason. Before initiating the study, the approval of the institutional review board committee was obtained. The teeth were inspected by naked eye, and any carious or malformed teeth were excluded. Teeth were thoroughly cleaned of debris and refrigerated in $0.1 \%$ thymol until being used within 1 month after extraction. ${ }^{10}$ Buccal or lingual surfaces of teeth were examined using a DIAGNOdent ${ }^{\circledR}$ pen. Forty-five specimens were prepared from those surfaces showing a reading less than 7 , while surfaces showing reading more than 7 were excluded. The sample size was calculated using OpenEpi, version 3 , open source calculator, SS mean. ${ }^{11}$

\section{Specimen Preparation}

The teeth were mounted from the root portion in self-cure acrylic resin blocks showing the crown only, the selected buccal or lingual surfaces were sectioned mesiodistally in approximately $2 \mathrm{~mm}$ thickness from the cusp tip using a diamond water-cooled diamond saw. ${ }^{12}$

A polyvinyl stencil of $4 \times 4 \mathrm{~mm}$ dimension was placed on the specimen surface. The surface was coated with a transparent acid-resistant nail varnish (REVLON, Oxford, UK). The stencil was removed after the set of the varnish, leaving a $4 \times 4 \mathrm{~mm}$ window on the surface. ${ }^{13}$ In this area, the artificial caries lesion was developed, assessed using the DIAGNOdent ${ }^{\circledR}$ pen and scanned by SEM.

A total of 45 specimens were individually separated in plastic test tubes and randomly assigned to three groups of 15 specimens each: in Group I (control), there was no treatment; Group II was treated by Clinpro ${ }^{\mathrm{TM}}$ white fluoride varnish containing $5 \%$ sodium fluoride $(22,600$ ppm fluoride ion) and innovative tricalcium phosphate ingredient, ethyl alcohol, n-hexane, thickener, and sweetened with xylitol (3M ESPE, USA), while Group III was treated with nano-HAP paste (Desensibilize Nano $\mathrm{P}$, Brazil) containing calcium nanophosphate organized in crystalline form of HA, potassium nitrate, water, surfactant, tensoative, flavor, and 9000 ppm sodium fluoride.

\section{Lesion Formation}

Each specimen was placed in continuously stirring incubator at $37^{\circ} \mathrm{C}$ and immersed in $8 \mathrm{~mL}$ of demineralizing solution for 4 days (96 hours). The solution was prepared using the formula $2.2 \mathrm{mM} \mathrm{CaCl}, 2.2 \mathrm{mM} \mathrm{KH}_{2} \mathrm{PO}_{4}$, and $0.05 \mathrm{M}$ acetic acid. $\mathrm{KOH}(1 \mathrm{M})$ was used to adjust $\mathrm{pH}$ to 4.4. ${ }^{14}$

The solutions were changed every day to avoid aggregation of demineralization products and $\mathrm{pH}$ change. After 96 hours, every specimen was washed thoroughly using deionized water and placed back in their test tubes immersed in deionized water until it was either measured by the DIAGNOdent pen or SEM.

\section{REMINERALIZATION}

Both fluoride varnish and nano-HAP paste were applied using $1 \mathrm{~cm}^{3} \mathrm{BD}^{\mathrm{TM}} \mathrm{U}-100$ insulin syringe (BD, Franklin Lakes, New Jersey, USA) to standardize the amount of agent applied on the lesion surface. A quantity of $0.02 \mathrm{~cm}^{3}$ of both agents was sufficient to cover the lesion surface of the specimens. ${ }^{6}$

In Group II, fluoride varnish was applied and airdried for 30 seconds. The specimens were stored in the remineralizing solution. After 4 hours, the varnish was removed with a scalpel blade gently to avoid touching the lesion surface. The surface of every specimen was washed using a syringe containing the remineralization solution: $1.5 \mathrm{mM} \mathrm{CaCl}_{2}, 0.9 \mathrm{mM} \mathrm{NaH} \mathrm{PO}_{4}$, and $0.15 \mathrm{M}$ $\mathrm{KCl}$ at $\mathrm{pH}$ of $7 .^{14}$

In Group III, according to the manufacturer's instructions, nano-HAP paste was applied on the specimen followed by friction of 10 seconds using a microbrush, then it was left in contact with the lesion surface for 4 minutes and removed with deionized water. This was done once a day for 2 days separated by one $\mathrm{pH}$ cycle.

\section{pH Cycling}

Standard $\mathrm{pH}$-cycling regimen was used to mimic the $\mathrm{pH}$ dynamics of the oral environment. It included 3 hours of demineralization and 21 hours of remineralization for 7 consecutive days. 
Table 1: DIAGNOdent ${ }^{\circledR}$ pen values interpretation

\begin{tabular}{ll}
\hline Value & Inference \\
\hline $0-13$ & Healthy tooth structure \\
$14-20$ & Enamel caries \\
$21-29$ & Deep enamel caries \\
$30+$ & Dentin caries \\
\hline
\end{tabular}

\section{DIAGNOdent ${ }^{\circledR}$ Pen Analysis}

The examination of the specimens was done using DIAGNOdent ${ }^{\circledR}$ pen to quantify the white lesions objectively. The instrument was calibrated against its own ceramic disk provided by the manufacturer before every measurement session. The instrument probe B was used in this study as it is indicated for smooth surface caries' detection.

The specimens were placed horizontally with the lesion facing upward, which allowed the standardization of the measurement by holding the tip $90^{\circ}$ to the lesion surface.

Measurements were interpreted according to the manufacturer of the DIAGNOdent ${ }^{\circledR}$ pen (Table 1). However, for research purpose and for inclusion phase, values measuring $<9$ were interpreted as shown in Table 2. ${ }^{12}$ Measurements were done at four stages: Baseline, after lesion formation, immediately after remineralization phase (posttreatment), and after 7 days of $\mathrm{pH}$ cycling.

\section{Scanning Electron Microscope Evaluation}

Scanning electron microscope analysis was carried out to qualitatively analyze the surface morphology of the specimens at the baseline, after demineralization, posttreatment, and after $\mathrm{pH}$ cycling. The randomly assigned specimens were air-dried and sputter-coated with gold under vacuum using Cressington 108 auto-sputter coater (Cressington Scientific Instruments Ltd., UK) and then examined under SEM at $\times 1,000$ magnification (Seron, Korea).

\section{Statistical Analysis}

Statistical analyses were performed using SPSS (Windows, version 20, Chicago, Illinois, USA). The alpha error was set at 0.05 . Kolmogorov-Smirnov tests were carried out to assess the normal distribution of continuous variables. Nonparametric tests were used for statistical comparisons because variables were not normally distributed.

Friedman tests followed by Wilcoxon tests were performed to compare the degree of remineralization and demineralization over time in each treatment group. Kruskal-Wallis tests followed by Mann-Whitney tests were performed to compare the three groups.
Table 2: DIAGNOdent ${ }^{\circledR}$ pen $<9$ values interpretation

\begin{tabular}{ll}
\hline Moment value & Inference \\
\hline $3-7$ & Normal \\
$7-9$ & Demineralization \\
$>9$ & White spot lesion \\
\hline
\end{tabular}

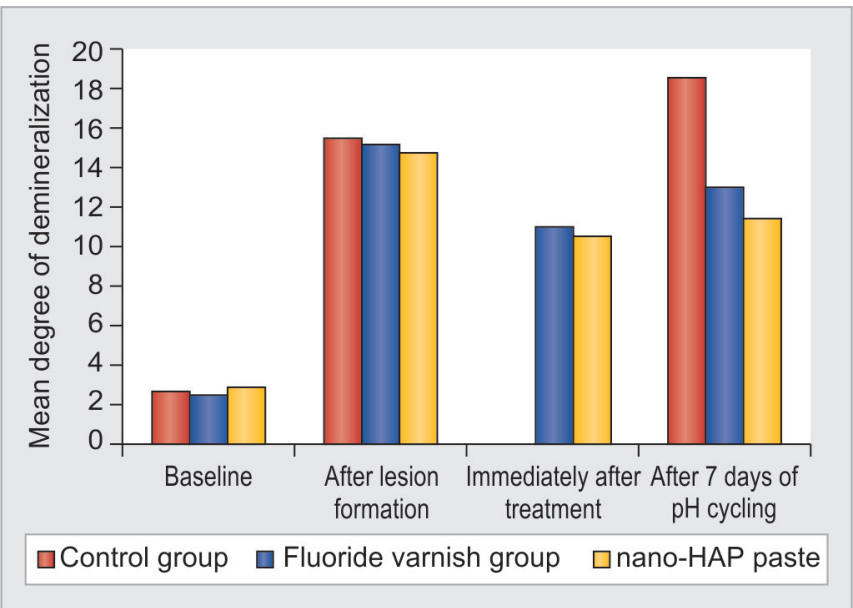

Graph 1: Mean degree of demineralization for the three groups at different quantitative assessment stages

\section{RESULTS \\ DIAGNOdent ${ }^{\circledR}$ Pen}

At baseline, the mean values were not significantly different between the three groups $(p=0.297)($ Graph 1$)$. Moreover, there was no significant difference between the three groups after lesion formation $(p=0.816)$. After treatment, there was no significant difference between the fluoride varnish group and the nano-HAP paste group $(\mathrm{p}=0.834)$. After $\mathrm{pH}$ cycling, a significant difference was found between the three groups $(p<0.001)$, where the degree of demineralization was significantly elevated in control group $(p<0.001)$; however, no significant difference was found between fluoride varnish group and nano-HAP paste group ( $\mathrm{p}=0.171$; Mann-Whitney test).

\section{Scanning Electron Microscope}

The SEM observation at $\times 1,000$ magnification showed smooth and intact surface at the baseline (Fig. 1). In Group I, after lesion formation, there was an increase in the surface porosity (Fig. 2A). After $\mathrm{pH}$ cycling, a marked increase in the porosities was evident (Fig. 2B). In Group II, SEM showed an evidence of reduction in the surface porosity (remineralization) (Figs 3A and B). However, after $\mathrm{pH}$ cycling, certain areas of porosity reappeared (Fig. 3C). In Group III, SEM showed a decrease in the porosity with deposition of nano-HAP crystals (Figs 4A and B). While, after $\mathrm{pH}$ cycling, nano-HAP crystals still could be observed with a smoother surface than that of fluoride group (Fig. 4C). 


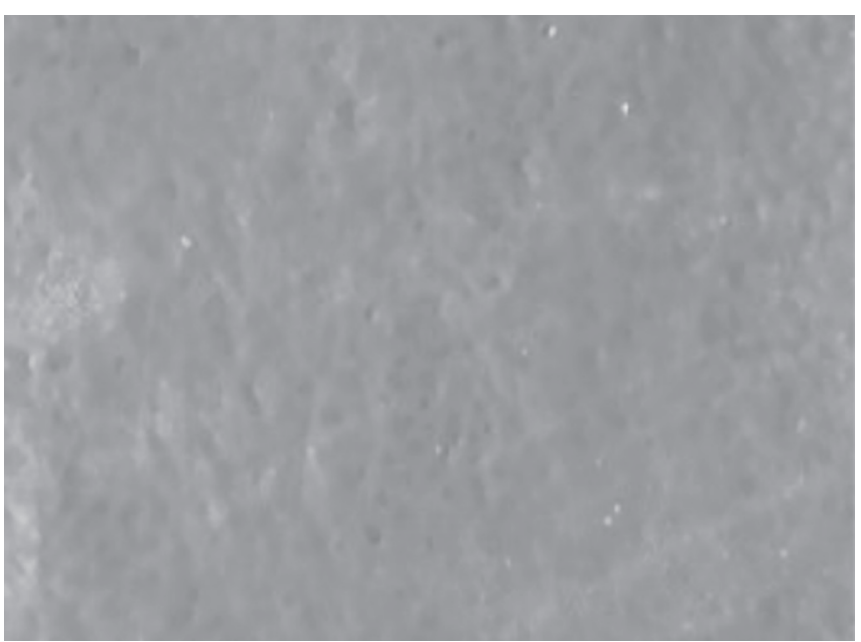

Fig. 1: Normal enamel at $\times 1,000$ magnification

\section{DISCUSSION}

Remineralization of noncavitated caries lesions noninvasively is one of the goals of modern noninvasive dentistry. ${ }^{1}$ Nano-sized HA provides high levels of biomimetic properties by its structure, composition, and physiochemical properties, which, in turn, could represent the key element for biomineralization of dental enamel. In recent years, the addition of nano-HAP to toothpastes and mouthwashes has shown some potentiality in remineralizing artificial demineralized lesions. ${ }^{9}$ The present study aims to compare the effect of a newly introduced chairside professional topical paste containing nano-HAP crystals with fluoride to fluoride varnish in remineralizing initial enamel lesion.

The sample size was calculated by comparing the fluoride varnish and nano-HAP paste Knoop microhardness $(\mathrm{KNH})$ mean values $(183.7 \pm 29.0,150.7 \pm$ 30.5 respectively), which represent one of two studies done on desensibilized nano-P paste. ${ }^{15}$ Minimal sample size of 12 in each group was needed to detect a difference with a power of $80 \%$ at $95 \%$ confidence level. Moreover, $10 \%$ was added to the sample size to cover the SEM samples which were lost through the treatment protocol.

In this study, two applications of nano-P paste were applied to simulate two professional applications, whereby the maximum remineralizing effect could be observed according to the manufacturer instructions. The fluoride varnish was applied once, similar to other in vitro studies. ${ }^{16-18}$ The specimens were left for 4 hours in the remineralizing solution before the removal of the fluoride varnish to stimulate clinical application protocol
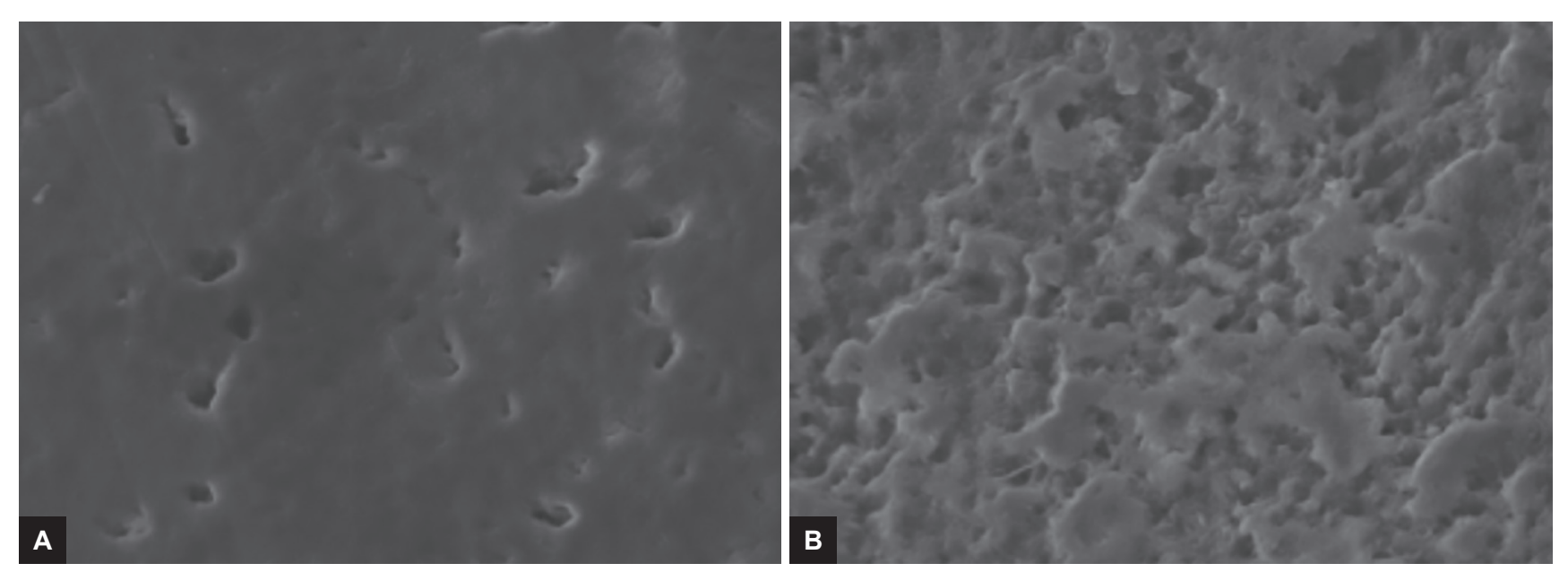

Figs 2A and B: Group I: Control group (A) after lesion formation; and (B) after pH cycling
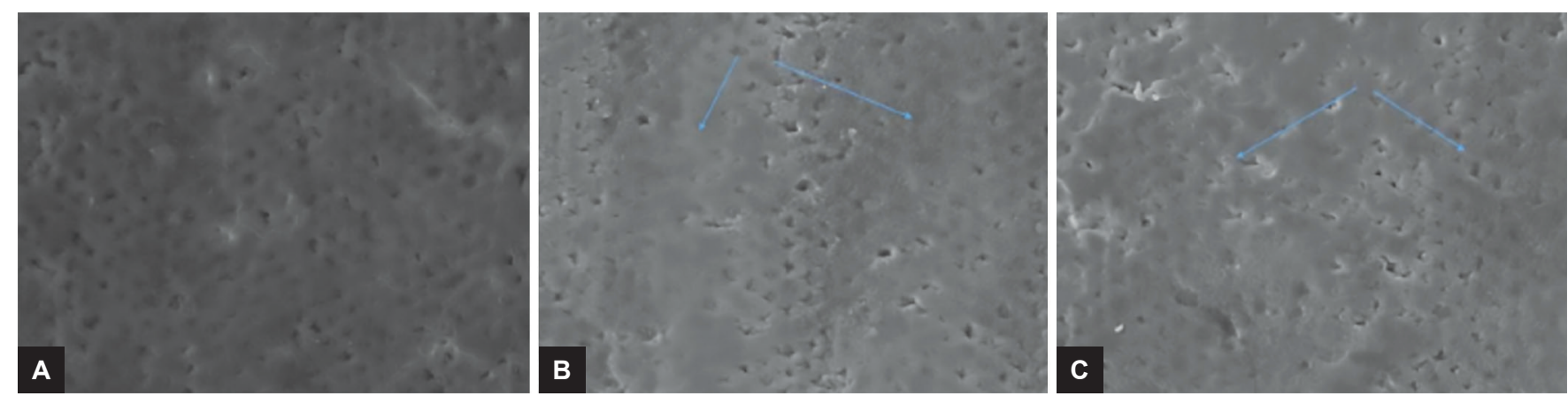

Figs 3A to C: Group II (fluoride varnish): (A) After lesion formation; (B) immediately after treatment with the arrows marking the evidence of reduction in the surface porosity; and $(\mathrm{C})$ after $\mathrm{pH}$ cycling, certain areas of porosity reappeared 

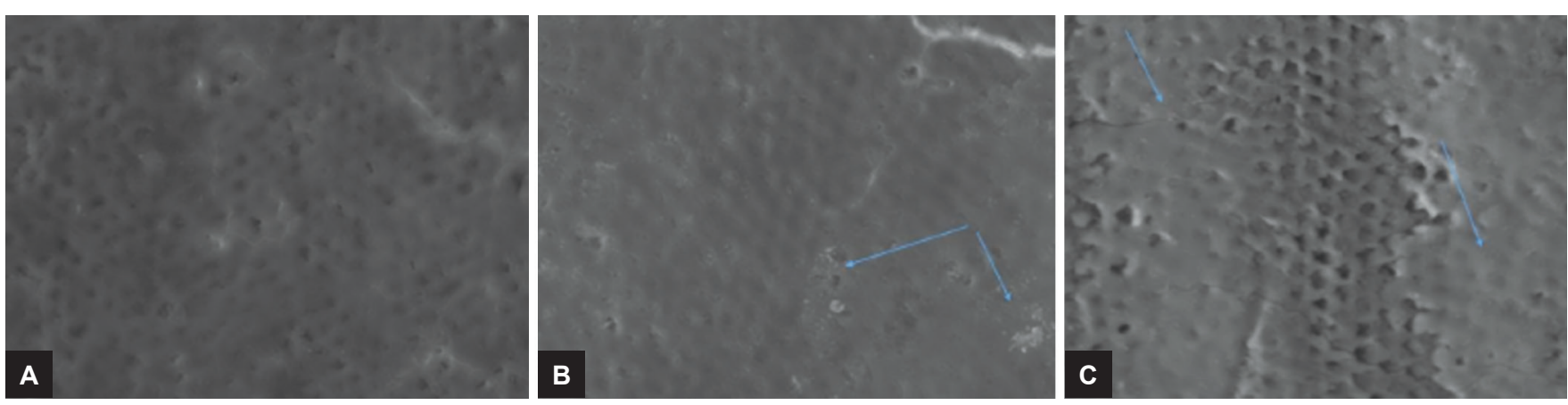

Figs 4A to C: Group III (Nano-HAP paste): (A) After lesion formation; (B) immediately after treatment with the arrows marking deposition of nano-HAP crystals; and (C) after 7 days of $\mathrm{pH}$ cycling, the nano-HAP crystals still could be observed

Table 3: Mean values and standard deviation of quantitative assessment of the degree of demineralization at different stages

\begin{tabular}{lllll}
\hline & Baseline & After lesion formation & Immediately after & treatment \\
\hline Control group $(\mathrm{n}=13)$ & $2.85 \pm 0.689^{\mathrm{a}}$ & $15.54 \pm 1.506^{\mathrm{a}}$ & - & After pH cycling \\
Fluoride varnish group $(\mathrm{n}=12)$ & $2.58 \pm 0.669^{\mathrm{a}}$ & $15.25 \pm 2.379^{\mathrm{a}}$ & $11.17 \pm 2.290$ & $18.62 \pm 2.063^{\mathrm{b}}$ \\
Nano-HAP group $(\mathrm{n}=12)$ & $3.00 \pm 0.953^{\mathrm{a}}$ & $14.83 \pm 2.250^{\mathrm{a}}$ & $10.67 \pm 2.535$ & $13.08 \pm 2.678^{\mathrm{a}}$ \\
Significance & 0.297 & 0.816 & 0.834 & $11.50 \pm 2.067^{\mathrm{a}}$ \\
\hline
\end{tabular}

${ }_{\mathrm{a}, \mathrm{b}}$ Mean of the multiple comparison tests. The same letter means no significant difference. The letter "a" means the smallest, etc.

where the patients are asked not to eat, brush, or floss for a minimal of 4 hours to prevent removal of the material from the tooth surface. ${ }^{19}$

In this study, the DIAGNOdent ${ }^{\circledR}$ pen was used as a noninvasive method that could give a quantitative assessment for the degree of demineralization. Healthy tooth structure displays little or no fluorescence, resulting in very low readings. However, when a demineralized area is present, the fluorescence will increase, which is indicated by the increase in the audible sound. Higher pitched sounds and higher number of sounds indicate more demineralization. ${ }^{12}$ The efficacy and the reproducibility of the DIAGNOdent ${ }^{\circledR}$ have been investigated by several studies. ${ }^{19,20}$ Moreover, DIAGNOdent ${ }^{\circledR}$ is found to be capable of diagnosing dental caries that were neither visible clinically nor radiographically. ${ }^{21,22}$

The results of this study showed that there was no significant difference between the three groups in the degree of demineralization at baseline (Table 3). Furthermore, there was no significant difference between the three groups after lesion formation. This indicates that standardized uniform artificial enamel lesions were obtained using the demineralization solution which made it possible to establish objective comparison among groups.

Immediately after treatment, both nano-P paste and fluoride varnish showed a significant decrease in demineralization with no significant difference between them. This showed that nano-HAP paste had a similar effect to fluoride varnish despite the fact that nano-HAP paste was left in contact with the specimen surface for a total of 8 minutes only and contained a lower fluoride concentration. Meanwhile, the fluoride varnish was left in contact with the specimen surface for 4 hours with higher fluoride concentration. This can be attributed to nano-HAP's high affinity and attachment onto enamel surface as suggested by several studies. ${ }^{23-25}$ The bioactive and biocompatible properties increased the potential of the nano-HAP to directly fill up defects and micropores on the demineralized enamel.

After $\mathrm{pH}$ cycling, there was a significant difference between the three groups, and the degree of demineralization was significantly elevated in control group (Table 3). However, there was no significant difference between the nano-P paste and fluoride varnish groups. Medeiros et $\mathrm{ll}^{15}$ investigated the performance of a single application of nano-P paste and fluoride varnish in the prevention of enamel erosion. Similar to our results, they found no significant difference among nano-P paste and fluoride varnish groups as assessed by surface $\mathrm{KNH}$. Nevertheless, they observed that nano-P paste showed the formation of a protective layer on enamel surface by atomic force microscopy, which, to some extent, simulate the cluster deposition of nano-HAP crystals found in our study using SEM. The efficiency of nano-P paste under dynamic $\mathrm{pH}$ cycling may be referred to the explicit filling and adherence of globular deposits of nano-HAP crystals in the enamel porosities which was observed by the SEM evaluation as shown in Figure 4. Nano-HAP crystals might have attracted a larger amount of $\mathrm{Ca}^{2+}$ and $\mathrm{PO}_{4}{ }^{3-}$ from the remineralizing agent which acted as a reservoir during $\mathrm{pH}$ cycling. ${ }^{9}$

On the contrary, de Carvalho et $\mathrm{al}^{6}$ reported that nano-HAP group showed significantly higher difference 
in surface hardness recovery after using the same $\mathrm{pH}$ cycling method as this study than fluoride varnish. This may be attributed to the number of applications of the nano-P paste, where it was applied for a total of seven times, while the fluoride varnish was applied only once. Taking into consideration the difference in the assessment method as they used $\mathrm{KNH}$, it can be deduced that increasing the times of application of the nano-P paste could enhance the effect of remineralization.

Scanning electron microscope analysis of the surface after remineralization induced by nano-HAP paste showed cluster depositions of nano-HAP crystals posttreatment, which was also noticed after $\mathrm{pH}$ cycling, this indicates that the adherence of the crystals was stable. These observations were in accordance with de Carvalho et $\mathrm{al}_{1}{ }^{6}$ who found similar cluster deposition using the same nano-HAP paste. In addition, similar results were observed by Swarup and $\operatorname{Rao}^{26}$ and Vyavhare et al, ${ }^{9}$ who studied the effect of adding synthetically processed nanoHAP to toothpaste in remineralizing initial enamel lesion.

In the fluoride varnish group, immediately posttreatment SEM showed a reduction in the surface porosity with areas of smooth enamel surface; however, an evidence of uncovered porous surfaces was observed after $\mathrm{pH}$ cycling. This result was not in accordance with the study of de Carvalho et al, ${ }^{6}$ who reported the presence of a homogeneous protective layer after $\mathrm{pH}$ cycling. This might be due to the variation of contact hours between the fluoride varnish and the tooth structure where the fluoride was applied for 4 hours in this study, while in de Carvalho et $\mathrm{al}^{6}$ study, it was applied for 24 hours, thus 4 hours might have been not enough to affect the surface morphology, where the effect of fluoride varnishes depends on the prolong contact of a thin layer which acts as a slow-release reservoir of fluoride. ${ }^{27}$

\section{CONCLUSION}

Within the limitation of this study, we can conclude that nano-HAP paste had similar potential in remineralizing initial enamel lesion as fluoride varnish. Moreover, under dynamic $\mathrm{pH}$ cycling conditions, nano-HAP paste showed promising results in surface depositions and maintaining a smooth surface over a longer duration.

\section{CLINICAL SIGNIFICANCE}

Based on the findings of this study, nano-HAP paste might be recommended as alternative remineralizing agent with lower fluoride concentration than fluoride varnish that could be beneficial for children, pregnant females, and those who are at high risk of dental fluorosis. Further in vivo investigations to assess the clinical performance of nano-HAP paste should be done.

\section{REFERENCES}

1. Abbas HM, Hamza HM, Ahmed HM. Minimal intervention approaches in remineralizing early carious lesions. J Am Sci 2012;8(3):709-717.

2. Acharya G, Agrawal P, Patri G. Recent biomimetic advances in rebuilding lost enamel structure. J Int Oral Health 2016 Feb;8(4):527-535.

3. Li X, Wang J, Joiner A, Chang J. The remineralisation of enamel: a review of the literature. J Dent 2014 Jun;42(Suppl 1): S12-S20.

4. Pepla E, Besharat LK, Palaia G, Tenore G, Migliau G. Nanohydroxyapatite and its applications in preventive, restorative and regenerative dentistry: a review of literature. Ann Stomatol (Roma) 2014 Jul-Sep;5(3):108-114.

5. Kantharia N, Naik S, Apte S, Kheur M, Kheur S, Kale B. Nanohydroxyapatite and its contemporary applications. JDRSD 2014 Jan;1(1):15-19.

6. de Carvalho FG, Vieira BR, Santos RL, Carlo HL, Lopes PQ, de Lima BA. In vitro effects of nano-hydroxyapatite paste on initial enamel carious lesions. Pediatr Dent 2014 May-Jun; 36(3):85-89.

7. Huang S, Gao S, Cheng L, Yu H. Combined effects of nanohydroxyapatite and Galla Chinensis on remineralisation of initial enamel lesion in vitro. J Dent 2010 Oct;38(10):811-819.

8. Huang S, Gao S, Cheng L, Yu H. Remineralization potential of nano-hydroxyapatite on initial enamel lesions: an in vitro study. Caries Res 2011 Sep;45(5):460-468.

9. Vyavhare S, Sharma DS, Kulkarni VK. Effect of three different pastes on remineralization of initial enamel lesion: an in vitro study. J Clin Pediatr Dent 2015 Winter;39(2):149-160.

10. Soe, MM.; Sullivan, KM. OpenEpi - toolkit shell for developing new applications. Atlanta (GA): Emory University; 2017. [cited 2017 May 30]. Available from: http://www.Openepi. com.; http:/ /www.openepi.com/SampleSize/SSMean.htm.

11. Huang SB, Gao SS, Yu HY. Effect of nano-hydroxyapatite concentration on remineralization of initial enamel lesion in vitro. Biomed Mater 2009 Jun;4(3):034104.

12. Jayarajan J, Janardhanam P, Jayakumar P, Deepika Efficacy of CPP-ACP and CPP-ACPF on enamel remineralizationan in vitro study using scanning electron microscope and DIAGNOdent. Indian J Dent Res 2011 Jan-Feb;22(1):77-82.

13. Mehta R, Nandlal B, Prashanth S. Comparative evaluation of remineralization potential of casein phosphopeptideamorphous calcium phosphate and casein phosphopeptideamorphous calcium phosphate fluoride on artificial enamel white spot lesion: an in vitro light fluorescence study. Indian J Dent Res 2013 Nov-Dec;24(6):681-689.

14. Watanabe MM, Rodrigues JA, Marchi GM, Ambrosano GM. In vitro cariostatic effect of whitening toothpastes in human dental enamel-microhardness evaluation. Quintessence Int 2005 Jun;36(6):467-473.

15. Medeiros IC, Brasil VL, Carlo HL, Santos RL, De Lima BA, De Carvalho FG. In vitro effect of calcium nanophosphate and high-concentrated fluoride agents on enamel erosion: an AFM study. Int J Paediatr Dent 2014 May;24(3):168-174.

16. Bolis C, Härtli GP, Lendenmann U. Fluoride varnishes-is there a correlation between fluoride release and deposition on enamel? Oral Health Prev Dent 2015 Jun;13(6):545-556.

17. Duraisamy V, Xavier A, Nayak UA, Reddy V, Rao AP. An in vitro evaluation of the demineralization inhibitory effect of F-varnish and casein phosphopeptide-amorphous calcium 
phosphate on enamel in young permanent teeth. J Pharm Bioallied Sci 2015 Aug;7(Suppl 2):S513-S517.

18. Rirattanapong P, Vongsavan K, Saengsirinavin C,Pornmahala T. Effect of fluoride varnishes containing different calcium phosphate sources on mineralization of initial primary enamel lesions. Southeast Asian J Trop Med Public Health 2014 Nov;45(6):1503-1510.

19. Spiguel MH, Tovo MF, Kramer PF, Franco KS, Alves KM, Delbem AC. Evaluation of laser fluorescence in the monitoring of the initial stage of the de-/remineralization process: an in vitro and in situ study. Caries Res 2009 May;43(4):302-307.

20. Celiberti P, Leamari VM, Imparato JC, Braga MM, Mendes FM. In vitro ability of a laser fluorescence device in quantifying approximal caries lesions in primary molars. J Dent 2010 Aug;38(8):666-670.

21. Iwami Y, Shimizu A, Hayashi M, Takeshige F, Ebisu S. Relationship between colors of carious dentin and laser fluorescence evaluations in caries diagnosis. Dent Mater J 2006 Sep;25(3):584-590.
22. Aktan AM, Cebe MA, Ciftçi ME, Sirin Karaarslan E. A novel LED-based device for occlusal caries detection. Lasers Med Sci 2012 Nov;27(6):1157-1163.

23. Li L, Pan H, Tao J, Xu X, Mao C, Gu X, Tang R. Repair of enamel by using hydroxyapatite nanoparticles as the building blocks. J Mater Chem 2008 Jul;18:4079-4084.

24. Roveri N, Battistella E, Bianchi CL, Foltran I, Foresti E, Iafisco M, Lelli M, Naldoni A, Palazzo B, Rimondini L. Surface enamel remineralization: biomimetic apatite nanocrystals and fluoride ions different effects. J Nanomater 2009 Jan; 2009:1-9.

25. Najibfard K, Ramalingam K, Chedjieu I, Amaechi BT. Remineralization of early caries by a nano-hydroxyapatite dentifrice. J Clin Dent 2011 Jan;22(5):139-143.

26. Swarup JS, Rao A. Enamel surface remineralization: using synthetic nanohydroxyapatite. Contemp Clin Dent 2012 Oct;3(4):433-436.

27. Ramaswami N. Fluoride varnish: a primary prevention tool for dental caries. J Mich Dent Assoc 2008 Jan;90(1):44-47. 